\title{
BMJ Open Patient safety initiatives in obstetrics: a rapid review
}

To cite: Antony J, Zarin W, Pham B', et al. Patient safety initiatives in obstetrics: a rapid review. BMJ Open 2018;8:e020170. doi:10.1136/ bmjopen-2017-020170

- Prepublication history and additional material for this paper are available online. To view these files, please visit the journal online (http://dx.doi. org/10.1136/bmjopen-2017020170).

Received 18 0ctober 2017 Revised 6 March 2018 Accepted 16 May 2018

Check for updates

${ }^{1}$ Knowledge Translation Program, Li Ka Shing Knowledge Institute, St. Michael's Hospital, Toronto, Ontario, Canada

${ }^{2}$ Toronto Health Economics and Technology Assessment, University of Toronto, Toronto, Ontario, Canada

${ }^{3}$ WHO Kobe Center, World Health Organization, Kobe, Japan

${ }^{4}$ Department of Geriatric Medicine, University of Toronto, Toronto, Ontario, Canada ${ }^{5}$ Epidemiology Division, Dalla Lana School of Public Health, University of Toronto, Toronto, Ontario, Canada

Correspondence to

Dr Andrea C Tricco;

triccoa@smh.ca

\author{
Jesmin Antony, ${ }^{1}$ Wasifa Zarin, ${ }^{1}$ Ba' Pham,,${ }^{1,2}$ Vera Nincic, ${ }^{1}$ Roberta Cardoso, \\ John D Ivory, ${ }^{1}$ Marco Ghassemi, ${ }^{1}$ Sarah Louise Barber, ${ }^{3}$ Sharon E Straus, ${ }^{1,4}$ \\ Andrea C Tricco ${ }^{1,5}$
}

\begin{abstract}
Objectives This review was commissioned by WHO, South Africa-Country office because of an exponential increase in medical litigation claims related to patient safety in obstetrical care in the country. A rapid review was conducted to examine the effectiveness of quality improvement (QI) strategies on maternal and newborn patient safety outcomes, risk of litigation and burden of associated costs.
\end{abstract}

Design A rapid review of the literature was conducted to provide decision-makers with timely evidence. Medical and legal databases (eg, MEDLINE, Embase, LexisNexis Academic, etc) and reference lists of relevant studies were searched. Two reviewers independently performed study selection, abstracted data and appraised risk of bias. Results were summarised narratively.

Interventions We included randomised clinical trials (RCTs) of Ql strategies targeting health systems (eg, team changes) and healthcare providers (eg, clinician education) to improve the safety of women and their newborns. Eligible studies were limited to trials published in English between 2004 and 2015

Primary and secondary outcome measures RCTs reporting on patient safety outcomes (eg, stillbirths, mortality and caesarean sections), litigation claims and associated costs were included.

Results The search yielded 4793 citations, of which 10 RCTs met our eligibility criteria and provided information on over 500000 participants. The results are presented by $Q$ I strategy, which varied from one study to another. Studies including provider education alone (one RCT), provider education in combination with audit and feedback (two RCTs) or clinician reminders (one RCT), as well as provider education with patient education and audit and feedback (one RCT), reported some improvements to patient safety outcomes. None of the studies reported on litigation claims or the associated costs.

Conclusions Our results suggest that provider education and other QI strategy combinations targeting healthcare providers may improve the safety of women and their newborns during childbirth.

\section{INTRODUCTION}

The rising costs in healthcare delivery and safety concerns of patients due to medical errors and liability claims have resulted in the development of policies to promote patient safety in medical practice. ${ }^{1-4}$ An increase in
Strengths and limitations of this study

A rapid review was conducted to identify quality improvement strategies for obstetrical care with supporting evidence from randomised clinical trials published in English between 2004 and 2015; a key limitation of the current review is the streamlined search and inclusion criteria used to accommodate the 6-week timeline for our decision-makers.

- To ensure the relevance of our review, commissioners from WHO South Africa-Country office were engaged in defining the review scope, developing review questions, approving the protocol and literature search strategies, and identifying key messages.

- A comprehensive search of the medical and legal databases, websites and reference lists of relevant studies were performed within the review scope.

- Study selection, data abstraction and quality appraisal were performed by two independent reviewers to minimise subjectivity and random errors.

the number of medical litigation cases and related costs is especially apparent in the field of obstetrics. ${ }^{5-7}$ Clinicians and decision-makers working in obstetrical care recognise the need to ensure the safety of patients, and many professional organisations (eg, American College of Obstetricians and Gynecologists, National Health Service) have taken steps to make this a priority by evaluating current practices and introducing patient safety initiatives in their organisations. ${ }^{358}$ Implementation of patient safety initiatives, including quality improvement (QI) strategies, aims to reduce the occurrence of avoidable adverse events and improve the quality of care. ${ }^{89}$ QI strategies can target health systems (eg, team changes, case management), healthcare providers (eg, provider education, audit and feedback) and/or patients (eg, patient education, self-management). These strategies are typically complex interventions with interacting components involving various stakeholders and targeting more than one level of care. ${ }^{10}{ }^{11}$ The evaluation of the effectiveness of these complex interventions 
is challenging and as such, the impact of QI interventions on patient safety outcomes remains unclear.

A scoping review on medical liability reforms and QI strategies to improve litigation-related outcomes in obstetrics identified several case studies with favourable findings. ${ }^{12}$ Since these findings were primarily limited to case studies with small sample sizes, an examination of their effectiveness was not feasible. The current rapid review, therefore, aimed to examine the effectiveness of QI strategies on patient safety outcomes, medical litigation claims and the associated costs.

\section{METHODS}

\section{Commissioning agency}

Due to an exponential increase in litigation claims related to patient safety in obstetrical care in South Africa, WHO South Africa-Country Office commissioned a review of patient safety initiatives. In order to provide decision-makers with timely results, a rapid review approach was collectively agreed on with a 6 -week timeline for completion. Rapid reviews tailor the systematic review process to produce information that is relevant to decision-maker needs in an abbreviated period of time. ${ }^{13}$ The streamlined steps followed in this review included limiting: the study design to randomised clinical trials (RCTs), search dates to a period of 10 years and language of publication to English.

\section{Protocol}

A protocol for this review was developed in collaboration with the review commissioner and revised by the team systematic review methodologist (ACT) and clinician (SES) (online supplementary file 1; appendix A). The conduct and reporting of this review followed guidance from the Preferred Reporting Items for Systematic Reviews and Meta-Analyses statement (online supplementary file 2). ${ }^{14}$

\section{Eligibility criteria}

The following PICOST (population, intervention, comparator, outcome, study design, and other limits) eligibility criteria were developed a priori:

\section{Population}

Pregnant women and/or newborns receiving care from professional healthcare practitioners (eg, physician, nurse and midwife) were eligible for inclusion.

\section{Interventions}

Interventions with the goal of promoting or ensuring patient safety in obstetric care (full definitions are provided in online supplementary file 1; appendix B) were eligible for inclusion. The patient safety interventions (hereafter referred to as QI strategies) targeted health systems (eg, clinician reminders, team changes) and/or healthcare providers (eg, provider education, audit and feedback). Studies with interventions that only targeted patients (eg, patient education, self-management) or community health workers (eg, village leaders, volunteers) were excluded because WHO was interested in interventions that they could implement at the health system or healthcare provider levels.

\section{Comparators}

Other patient safety interventions or usual care were eligible comparators.

\section{Outcomes}

Adverse safety outcomes (eg, physical or mental damage or injury to the pregnant woman, fetus or newborn), litigation claims (eg, lawsuits or other legal action) and the associated costs (eg, cost of patient safety initiatives to reduce harms and litigation or expenditure due to medical adverse event or legal outcome) were eligible for inclusion. The following outcomes were selected by the clinician (SES) on the team and review commissioner as key safety outcomes of interest: stillbirths, perinatal mortality, neonatal morality, maternal mortality and caesarean sections. However, other patient safety outcomes (eg, neonatal morbidity, blood loss and haemorrhage) reported in the included studies were also reported.

\section{Study design}

Due to the rapid nature of the review, only RCTs, including cluster randomised trials, were included. Quasi-randomised trials and non-randomised studies were not eligible for inclusion.

\section{Other}

Additional limits to accommodate the 6-week timeline included publication date (ie, 2004-2015) and language of publication (ie, English only).

\section{Information sources and literature search}

An electronic search of the literature was conducted in MEDLINE, Embase, LexisNexis Academic, LegalTrac and the Legal Scholarship Network on 13 August 2015. The search was limited to RCTs (using a validated search filter) ${ }^{15}$ as well as papers published in English from 2004 to 2015 .

The MEDLINE search strategy was developed by an experienced librarian (McGowan) in consultation with the research team, approved by the review commissioner, and peer-reviewed by another librarian (Cogo) using the Peer Review of Electronic Search Strategies checklist. ${ }^{16}$ The final search strategy for MEDLINE can be found in online supplementary file 1 ; appendix $\mathrm{C}$, and was adapted for the other electronic databases. The bibliographic search was supplemented by searching websites of WHO (http://www.who.int/en/) and Canadian Medical Protective Association (https://www.cmpa-acpm.ca/en/ home) and scanning reference lists of all included RCTs.

\section{Study selection}

The search results were screened using our proprietary web-based tool, Synthesi.SR. ${ }^{17}$ The inclusion criteria and 
screening questionnaires were established a priori for screening of titles and abstracts, and full-text articles. To ensure inter-rater agreement, a random sample of 50 citations was pilot tested among the review team with $100 \%$ agreement across reviewers. The remaining search results were independently screened by pairs of reviewers (JA, WZ, VN, RC, JDI, MG, CW, MK, RW and SM) and discrepancies were resolved by a third reviewer (JA,WZ or ACT). The same process was followed for screening of potentially relevant full-text articles in which a pilot test was conducted on a random sample of 20 full-text articles with $90 \%$ agreement across reviewers.

\section{Data abstraction}

Data were collected for predefined sets of items using a standardised form in Excel. Data items included study characteristics (eg, author, country of conduct and study design), patient characteristics (eg, target population and sample size), description of the QI strategies (eg, provider education and team changes) and patient safety outcome results (eg, stillbirths, neonatal mortality, litigation cases and costs). The form was pilot tested on one article with a facilitated discussion to clarify discrepant items. Pairs of reviewers then abstracted data from each article, independently (JA, WZ, VN, RC, JDI and MG). Differences in abstraction were resolved by discussion and/or the involvement of a third team member (JA, WZ, VN, RC and ACT). The QI strategies used in each treatment arm were identified and categorised by an experienced systematic review methodologist (ACT) and clinician (SES) independently, and discrepancies were resolved through discussion.

\section{Risk of bias assessment}

Risk of bias of the included RCTs was assessed using the 7-item Cochrane Risk-of-Bias tool ${ }^{18}$ by pairs of reviewers independently (JA, WZ, VN, RC, JDI and MG). Since all reviewers were experienced with this tool, we did not conduct a pilot test. For the 'other bias' component of the tool, we assessed the potential for funding bias, as well as the presence of an imbalance in baseline numbers, risk of contamination and confounding bias due to differences in treatment administration as described by the authors of the included studies. Discrepancies were resolved by a third reviewer (JA, WZ or ACT).

\section{Synthesis}

Study, patient and intervention characteristics were summarised using descriptive analysis. All patient outcomes were synthesised narratively.

\section{Patient involvement}

No patients were involved in setting the research question or the outcome measures, nor were they involved in the design and implementation of the study.

\section{RESULTS}

The literature search resulted in 4793 citations (figure 1). After screening for eligibility based on titles and abstracts,
276 potentially relevant full-text articles were identified and screened for inclusion. Ten RCTs ${ }^{19-28}$ with one companion report ${ }^{29}$ met the inclusion criteria and were included.

\section{Study characteristics}

Although all RCTs were published from 2004 to 2015, they were conducted between the years of 1982 and 2011 with study durations ranging from $2^{19} 2426$ to 21 years ${ }^{27}$ (online supplementary file 1; appendix D). Over 500000 participants were included across the RCTs from North America $(\mathrm{n}=5),{ }^{21} 2426-28$ South America $(\mathrm{n}=2),{ }^{19}{ }^{20}$ Africa $(\mathrm{n}=2)^{22} 23$ and Australia $(\mathrm{n}=1) .{ }^{25}$ Two RCTs were randomised at the patient level (RCTs), ${ }^{25} 27$ while eight were cluster RCTs randomised at the obstetrics unit, hospital or district level. ${ }^{19-24} 2628$

\section{Patient characteristics}

Two RCTs described QI strategies targeting the health system, such as team changes and case management. One of these RCTs focused on QI strategies implemented for the improvement of outcomes in pregnant women alone, ${ }^{25}$ while the other involved the care of pregnant women and children up to 2 years of age ${ }^{27}$ (online supplementary file 1; appendix D). All cluster RCTs described QI strategies targeting healthcare providers, such as clinicians, nurses and midwives. ${ }^{19-24} 2628$ The intervention settings of the RCTs were hospitals $(n=8 ; 80 \%)$, and/or communities $(n=3 ; 30 \%)$.

\section{Risk of bias appraisal}

All 10 RCTs were assessed as having a low risk of ascertainment bias since the outcomes were examined using objective measures (eg, blood loss; figure 2). Seven RCTs $(70 \%)$ were assessed as having a low risk of bias for random sequence generation, as well as low risk of attrition bias. About half of the RCTs were considered to be either high or unclear risk of bias for allocation concealment and selective reporting. Three studies were assessed as having a high risk of 'other bias'21 2528 due to systemic between-group differences in the distribution of baseline characteristics, potential bias due to uneven implementation of the intervention in different clusters, and/or failing to assess or adjust for other confounders (eg, baseline risk of adverse pregnancy outcomes).

\section{Patient safety outcomes}

All RCTs reported on patient safety outcomes for mothers and their babies. In total, we identified 26 safety outcomes reported in the 10 studies. None of the 10 RCTs reported on outcomes related to litigation or associated costs. As each of the intervention components varied significantly, we were unable to statistically combine the results in a meaningful way using meta-analyses. Therefore, results were synthesised and summarised narratively. The findings of each study are presented below by intervention components. As a supplement to our results, detailed descriptions of each of the included interventions (online supplementary file 1; appendix E), definitions of key outcomes (online 

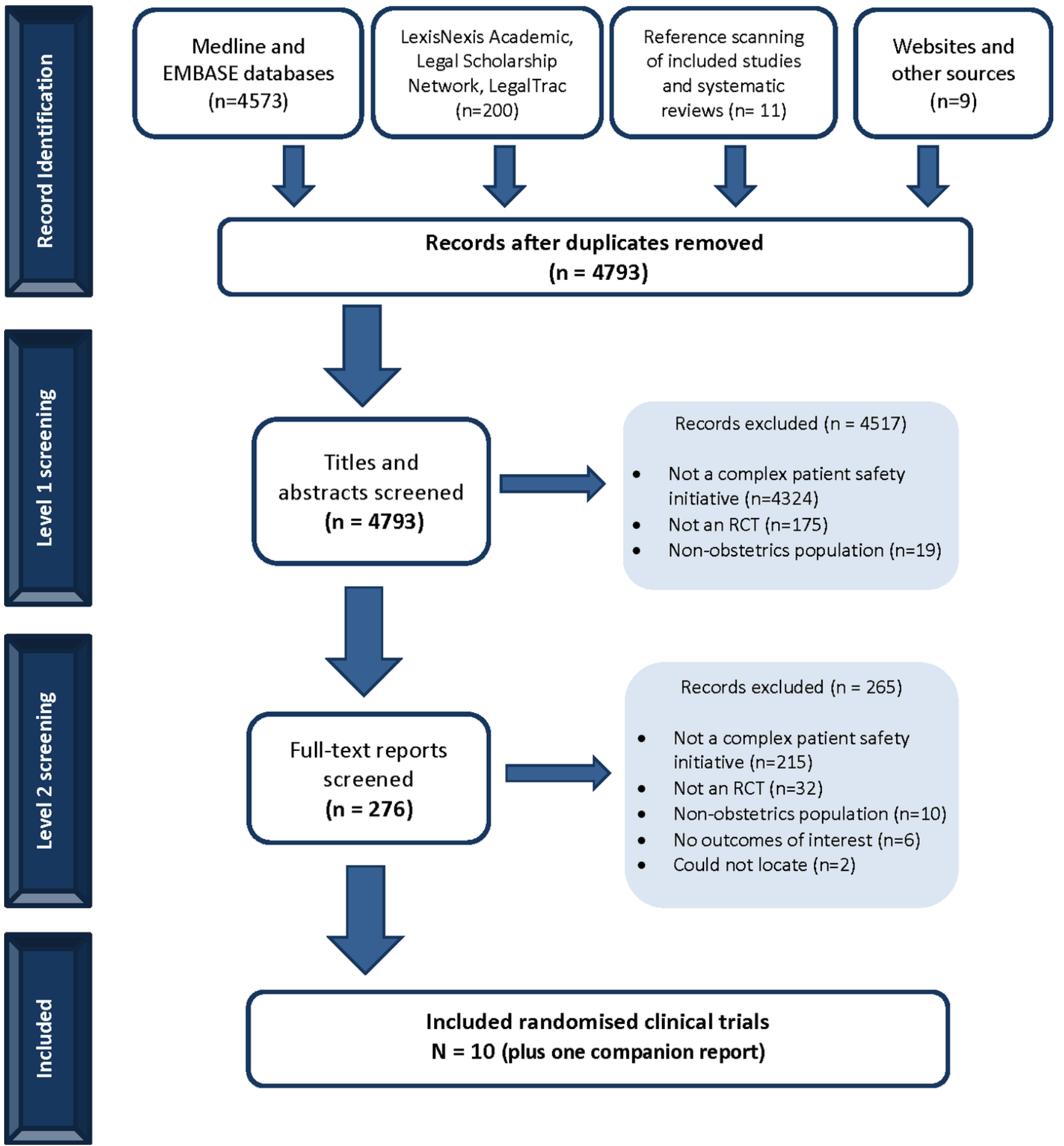

Included randomised clinical trials

Figure 1 Study flow diagram. Breakdown of the number of studies identified in the literature, assessed for eligibility, and finally included in the rapid review on patient safety initiatives in obstetrics. RCT, randomised clinical trial.

supplementary file 1; appendix F) and study-specific conclusions by outcome (table 1) are also presented.

\section{Provider education $(\mathrm{n}=2)$}

Althabe $e$ t $a l^{19}$ compared the use of a mandatory second opinion by a clinician trained to use a new decision-aid tool to usual care before caesarean section. This decision-aid tool provided clinicians with suggestions and recommendations on how to prevent non-emergency caesarean sections. This cluster RCT of 149276 pregnant women found a small significant reduction in the rate of caesarean section for the intervention versus usual care (relative rate reduction $7.3 \%, 95 \%$ CI 0.2 to 14.5 ). Other safety outcomes of maternal, perinatal and neonatal mortality, as well as unplanned admission to the neonatal intensive care (NICU) and intensive care unit (ICU) 


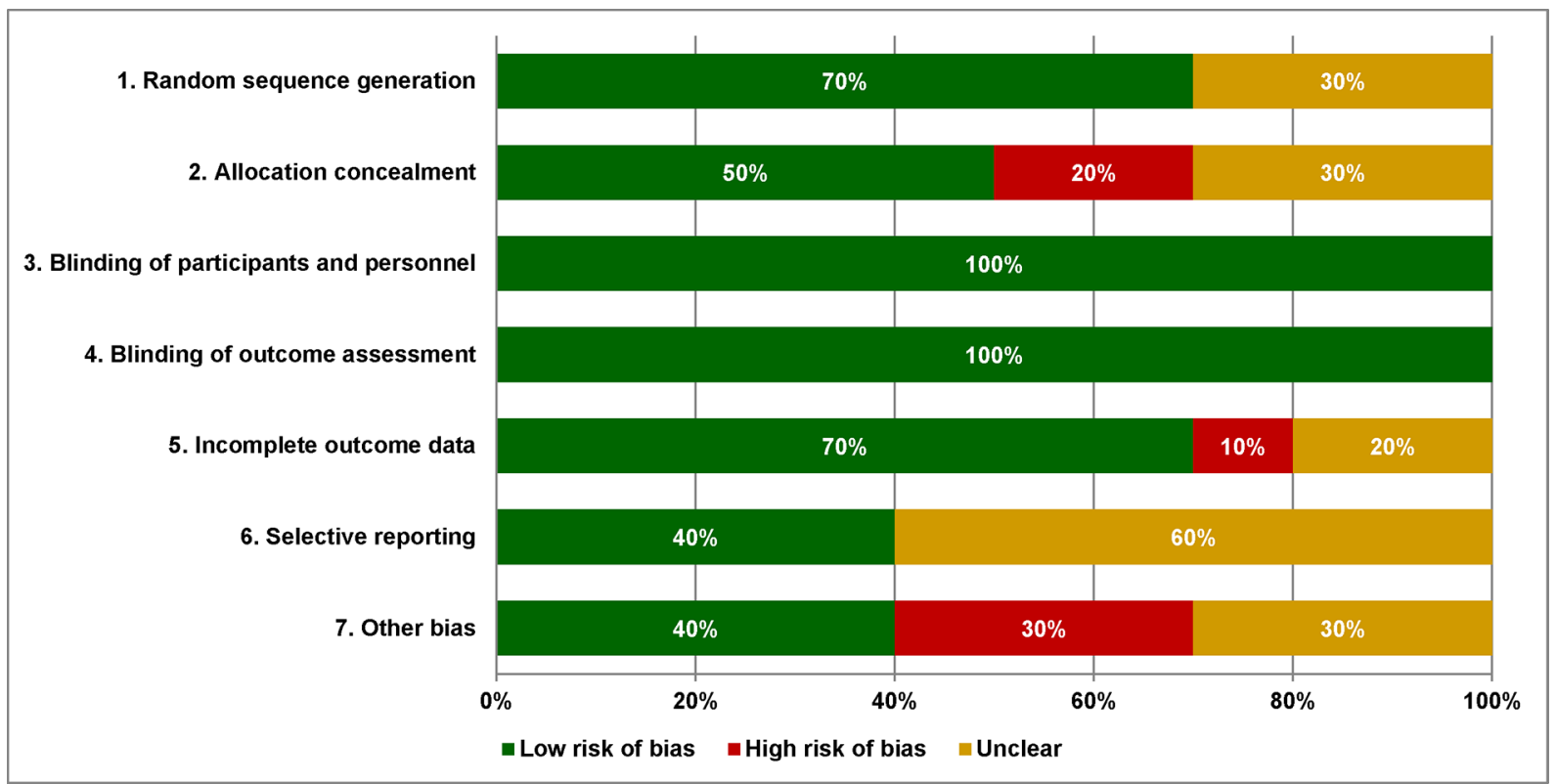

Figure 2 Risk of bias. Aggregate Cochrane risk-of-bias appraisal results.

showed no significant differences between groups. This RCT had an unclear risk of selective reporting bias and other bias.

The impact of team and staff training was evaluated in a cluster RCT published by Riley and colleagues. ${ }^{28}$ Three hospitals in the United States were compared in this RCT: one control hospital (no intervention), one hospital used didactic training only (based on an evidence-based teaching plan with a focus on leadership, situation monitoring, mutual support and communication) and one hospital received the full intervention (didactic training with patient simulations from triage through labour and recovery). The 4-year follow-up showed no statistically significant differences in the pre-intervention and post-intervention results in the hospitals administering the control and didactic programmes on the Weighted Adverse Outcome Score (WAOS) including 10 adverse outcomes. However, the hospital receiving the full intervention reported a statistically significant change in WAOS, suggesting that a complex intervention including didactic training with situational simulation can improve the safety of obstetrical patients. This RCT had an unclear risk of bias on random sequence generation, incomplete outcome reporting, selective reporting bias and allocation concealment.

Provider education with audit and feedback $(\mathrm{n}=2)$

A cluster RCT by Chaillet et $a l^{21}$ conducted across 32 hospitals in Quebec assessed the effect of a multifaceted strategy to promote professional onsite training (including staff education, educational outreach, as well as audit and feedback) on the number of caesarean deliveries and other maternal and neonatal outcomes. No intervention was administered to the 16 hospitals in the control arm. During the 2-year intervention and follow-up period, there were 184952 deliveries included. A small, statistically significant reduction in the number of caesarean births was observed in the intervention arm $(\mathrm{p}=0.04)$. The intervention group also had statistically significantly lower major neonatal morbidity $(\mathrm{p}=0.03)$ and a significantly smaller increase in minor neonatal morbidity $(p<0.001)$ when compared with the control group. There were no significant differences between groups in maternal morbidity. This RCT had a low risk of bias across all components except allocation concealment (unclear) and other risk of bias (high).

Dumont $e t a l^{23}$ reported the effects of a complex intervention in a cluster RCT conducted in Senegal and Mali. The intervention arm included an initial interactive workshop on evidence-based clinical practice and the clinical audit process attended by opinion leaders (physicians and midwives) from 23 hospitals. The trained opinion leaders then returned to their respective hospitals to launch maternal death audits and provide on-site training, including quarterly educational outreach visits. The control arm included 95236 patients in 23 hospitals that did not receive any intervention. Outcomes assessed at baseline and after 4 years of follow-up on a total of 191167 patients found that maternal death reviews and on-site training may be beneficial for certain populations. Compared with the control group, the intervention arm resulted in better maternal mortality rates (OR $0.85,95 \%$ CI 0.73 to 0.98 ), although this was limited to capital and district hospitals (where mild complications were managed as the first level of care, prior to the involvement of regional-level or national-level hospitals). This RCT was assessed as having a low risk of bias on all components except random sequence generation and allocation concealment, which were both scored as having an unclear risk of bias.

Provider education with clinician reminders $(n=1)$

Althabe $e t a l^{20}$ published a cluster-RCT exploring a multicomponent behavioural intervention to facilitate the 


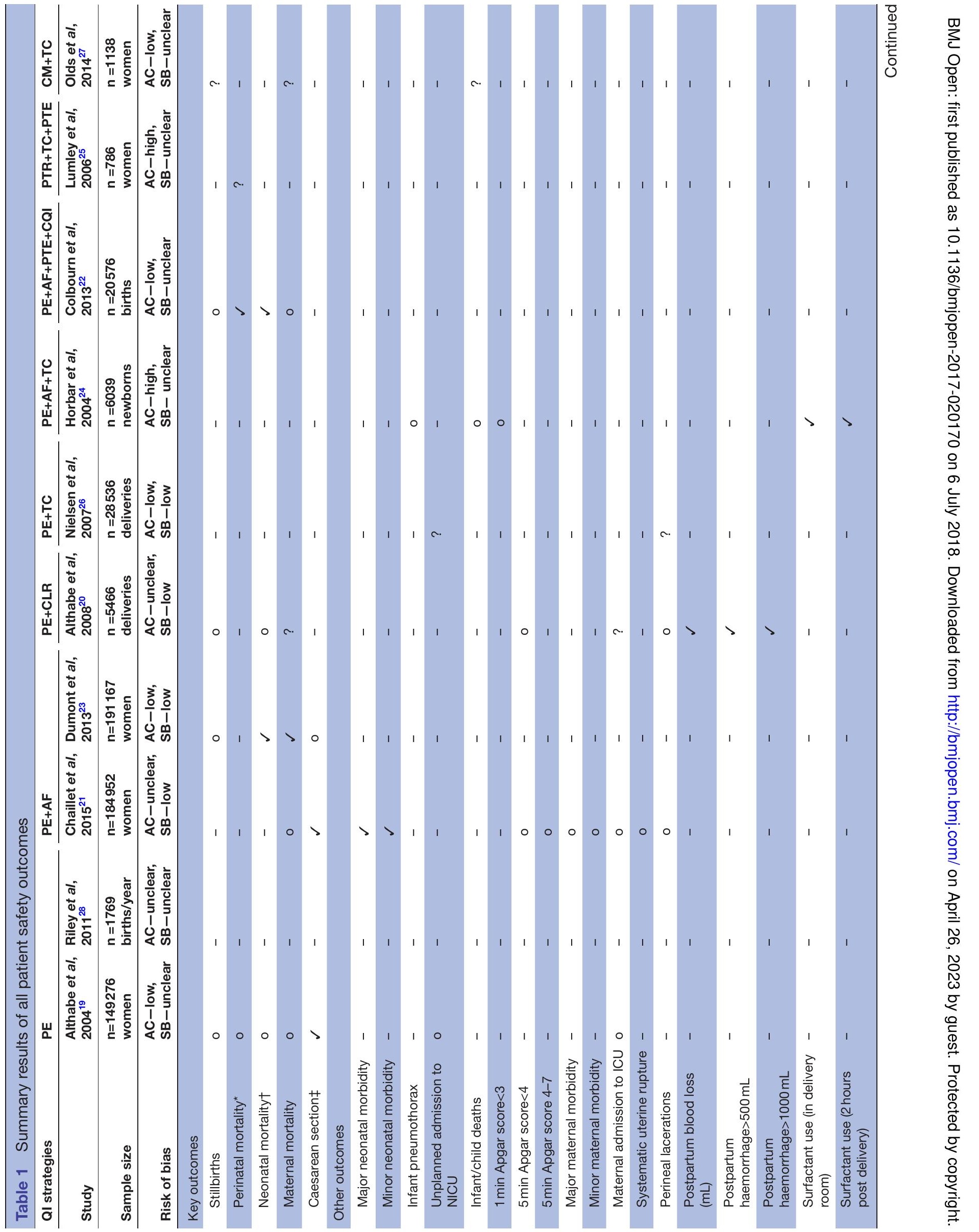



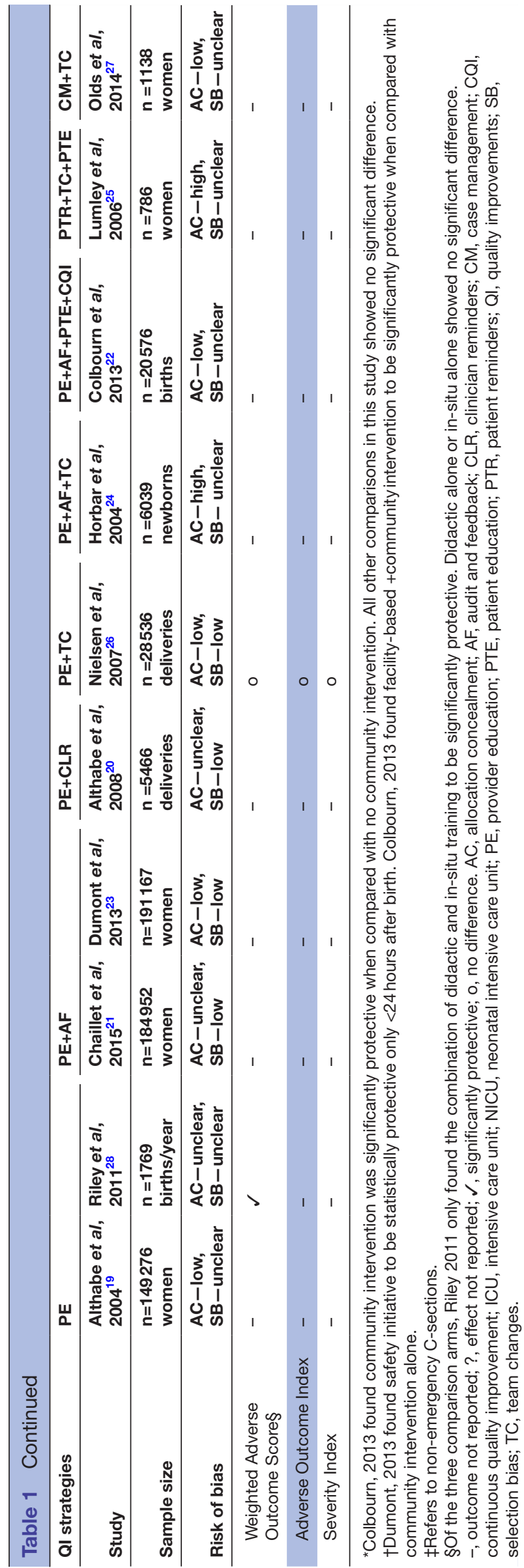

implementation of two evidence-based practices: the selective use of episiotomy and active management of the third stage of labour. The intervention involved the use of opinion leaders, staff training and staff reminders. Ten hospitals in Argentina and Uruguay reporting 2963 deliveries acted as the treatment arm. Nine hospitals with 2503 vaginal deliveries formed the control group and received no intervention besides the standard in-service training. The outcomes of interest were assessed at baseline and at 18 months. When looking specifically at the adverse events to patients, there was a statistically significant relative rate reduction in postpartum haemorrhage and blood loss in the intervention arm at $500 \mathrm{~mL}$ or more $(45 \%, 95 \%$ CI 9 to 71$)$ and $1000 \mathrm{~mL}$ or more $(70 \%, 95 \%$ CI 16 to 78$)$. Maternal death, maternal admission to the ICU, neonatal death, stillbirths or Apgar score $<4$ did not result in a significant difference. The RCT was assessed as having an unclear risk of bias associated with random sequence generation and allocation concealment.

Provider education with team changes $(n=1)$

Nielsen and colleagues ${ }^{26}$ evaluated the effect of staff teamwork training on adverse outcomes in labour and delivery units in the United States. Teamwork training was administered in two parts with selected staff attending training sessions on communication and team structure, and then returning to their home hospitals to train other staff members. Analysis was conducted on 28536 deliveries. The Adverse Outcome Index (AOI) was used to calculate the proportion of patients with one or more adverse outcomes. The WAOS was also used to consider the relative severity of the included adverse outcomes. Some of the adverse events considered in these scores included maternal death, neonatal death, uterine rupture, maternal admission to the ICU, unplanned admission to the NICU, Apgar score $<7$ and birth trauma. However, no statistically significant differences between groups were observed for the AOI, WAOS or any of the individual adverse outcomes assessed. The risk of bias for this RCT was deemed low for all items except other risk of bias, which was unclear.

\section{Provider education with audit and feedback and team changes $(\mathrm{n}=1)$}

The RCT by Horbar et $a t^{24}$ evaluated a multicomponent patient safety intervention to promote evidence-based surfactant treatment for preterm infants, including individualised audit and feedback cycles, education and training of staff, and collaboration among intervention arm teams. Fifty-seven hospitals administered the patient safety intervention, while another 57 hospitals acted as the control. The use of surfactant in the delivery room was significantly higher in the intervention group than the control group (adjusted OR 5.38, 95\% CI 2.84 to 10.20 ), while the intervention hospitals had significantly lower surfactant treatment more than 2 hours after birth when compared with the control hospitals (adjusted OR $0.35,95 \% \mathrm{CI} 0.24$ to 0.53 ). The other outcomes, including 
pneumothorax and infant mortality, were not found to be significantly different. The RCT had a high risk of bias with respect to allocation concealment and an unclear risk of selective outcome reporting bias.

Provider education with audit and feedback, patient education and continuous quality improvement $(\mathrm{n}=1)$

In rural Malawi, Colbourn et $a l^{22}$ conducted a two-by-two factorial cluster RCT examining the use of a women's group community intervention and a facility-based QI intervention to reduce maternal, perinatal and neonatal mortality. The first group received the community intervention consisting of patient education, the second group received facility-based provider education and audit and feedback, the third group received both community and facility-based interventions and the final group acted as a control arm. The analysis consisted of 5080 in the community group, 5335 in the facility group, 5249 in the combined group and 4912 infant births in the control group. The community intervention group alone had a significantly lower perinatal mortality rate ( $16 \%$ lower) when compared with control (adjusted OR 0.84, 95\% CI 0.72 to 0.97$)$. On the other hand, the neonatal mortality rate was $22 \%$ lower in the facility-based+community interventions combined compared with control (adjusted OR $0.78,95 \%$ CI 0.60 to 1.01 ). No significant effects were reported for maternal mortality. The RCT was assessed as having a low risk of bias on all items except selective outcome reporting, which was unclear.

\section{Patient reminders with team changes and patient education $(n=1)$} Lumley et $a l^{25}$ conducted an RCT in Australia to assess the impact of a prepregnancy advice/counselling service offered to new mothers (initiated by two obstetricians) on the well-being of their second-born children. There were 392 women in the intervention arm who were identified after the birth of their first child. These women worked with a midwife (ie, team changes) to identify current health and lifestyle problems, assess family/genetic history, receive education and referrals as needed, and discuss and develop an appropriate plan for their next pregnancy (including a reminder card). Meanwhile, 394 women in the control arm received a home visit with an opportunity to discuss their first pregnancy and ask questions. Outcomes were assessed after the birth of the second child. Infants born to mothers who received counselling were more likely to be of lower birth weight than those who did not, and there were no significant differences between the groups in secondary outcomes such as perinatal deaths and congenital malformations. The RCT had an unclear risk of selective reporting bias, and high risk of bias on the allocation concealment, incomplete outcome data and other bias items.

\section{Case management and team changes $(\mathrm{n}=1)$}

One RCT was conducted by Olds et al. ${ }^{27}$ to determine the effect of prenatal and infant home visits by nurses on maternal and child mortality. Participants, mostly African-American women residing in very poor neighbourhoods, were randomised to one of four treatment arms during pregnancy and were followed for 2 years. In treatment 1, 166 women received free transportation for prenatal appointments. In addition to transport, 514 women in treatment 2 also received some developmental screening and referral services. The third treatment arm including 230 women added nurse home visits during pregnancy as well as 2 postpartum home visits, while 228 women in treatment 4 received the most comprehensive intervention with transport, screenings, nurse home visits during pregnancy and until the child was 2 years old. Maternal and infant mortality outcomes were collected for all treatment arms after 2 years of follow-up. Participants in the combined control arm (treatment 1+treatment 2) had more natural, preventable and total infant deaths when compared with women receiving a combined intervention including treatment 3 and 4 . Survival curves were created for each of the treatment arms. When projecting to 21 years after randomisation, all-cause mortality in mothers was statistically significantly higher in treatment $1+$ treatment 2 when compared with treatment 3 alone $(p=0.007)$ or when compared with treatment $3+$ treatment $4(\mathrm{p}=0.008)$. The RCT was assessed as having an unclear risk of allocation concealment, incomplete outcome data and selective reporting bias.

\section{DISCUSSION}

We conducted a rapid review and identified 10 RCTs written in English and published between 2004 and 2015 on complex interventions that can be used to improve patient safety in obstetrics. The included RCTs examined a broad range of complex patient safety interventions in obstetrics with some treatment arms including only one QI strategy, while others were multifaceted interventions including up to four QI strategies. Many of the included studies had a provider education component and the results suggest that this intervention, when combined with other QI strategies, may improve outcomes. Results from two RCTs indicated that provider education with audit and feedback may improve patient safety, specifically by lowering neonatal morbidity and caesarean births, ${ }^{21}$ as well as neonatal and maternal mortality, ${ }^{23}$ when compared with usual care. In another RCT, patients receiving provider education combined with clinician reminders had reduced postpartum blood loss and haemorrhage when compared with control groups in similar settings. ${ }^{20}$ Finally, an RCT comparing the use of provider education with patient education and audit and feedback compared with community intervention alone demonstrated an improvement in patient safety through a reduction in neonatal mortality. ${ }^{22} \mathrm{~A}$ future comprehensive systematic review that considers quasi-experimental and observational study designs should be conducted on this topic to provide a definitive conclusion on whether these interventions are indeed effective. Such a systematic 
review may be able to include more studies, allowing the conduct of a meta-analysis of the QI strategies versus usual care and potentially quantifying the effectiveness of these interventions.

The quality of the included RCTs was generally high, with a few areas of concern. It was unclear whether randomisation sequence was sufficiently concealed, or whether selective outcome reporting was present, since these items were unclear for half of the included studies. Also, 6 out of 10 RCTs were graded as either 'unclear' or 'high risk of bias' for the 'other bias' category, as differences in baseline characteristics or confounding effects due to differences in treatment administration across providers, departments or hospitals were concerns reported by the study authors themselves.

A major strength of our review was the timely provision of high-quality evidence for decision-makers. Our rapid review methodology included a comprehensive search of the literature using multiple databases, and study selection, data abstraction and risk of bias assessment performed in duplicate by pairs of reviewers. However, as with any rapid review, there are also some limitations to be considered. We had to methodologically tailor our review to suit the decision-makers needs by limiting results to RCTs published in English within a 10-year timeframe. In addition, the literature search was conducted in August 2015 for the purpose of submitting a report to the review commissioners who did not request that we update our findings. Moreover, variation in administration and implementation of the QI strategies across settings are unavoidable, especially in cluster RCTs, where each hospital acts as an independent unit. Consideration should be made of possible confounding effects as a result of the hospital setting and care practices (eg, duration, frequency and provider). Given the number and range of patient safety initiatives included in each study, it is difficult to ascertain how each of the components included in the multifaceted, complex interventions directly contributed to the observed effects. Additionally, it was challenging to compare across studies as the QI strategies were used to address different clinical questions in each (eg, prenatal home visits by midwives to reduce preterm births compared with teamwork training in hospitals to promote guideline implementation). The differences in these complex interventions meant we were unable to conduct meta-analysis. Moreover, classifying complex interventions, such as QI strategies, is challenging ${ }^{30}$ and required two individuals with complementary expertise to conduct this task.

Finally, we did not identify any RCTs specifically addressing litigation claims or undue costs to the healthcare system. However, evidence from non-randomised studies suggests that there may be a relationship between a reduction in adverse safety outcomes and a reduction in litigation and losses due to medical errors and malpractice. These reports ${ }^{531}$ have found that the introduction of patient safety programmes, involving a combination of strategies targeting health systems and healthcare providers, have resulted in the reduction of obstetrical adverse events and the number of litigation claims and resulting costs. In addition, the community and facility-based interventions evaluated in the Colbourn et $a l^{22}$ trial were shown to be highly cost-effective in an economic evaluation conducted by the study authors. ${ }^{32}$ Further research is needed to examine the effectiveness and cost-effectiveness of patient safety interventions for adverse events, litigation claims and associated costs.

\section{CONCLUSIONS}

Our results suggest that provider education and other QI strategy combinations targeting healthcare providers may improve the safety of women and their newborns during childbirth. In addition, improved patient safety may influence the risk of medical litigation claims and associated costs; however, no direct evidence was found for these outcomes. A future systematic review, including a meta-analysis, may be able to provide more definitive conclusions.

Acknowledgements The authors thank Dr. Jessie McGowan for developing the literature searches, Elise Cogo for peer reviewing the literature searches and Alissa Epworth for executing the literature searches in the legal databases and obtaining the full-text articles. They also thank Charlotte Wilson, Meghan Kenny, Rachel Warren and Sanober Motiwala for assisting with level 1 and level 2 screening, as well as Inthuja Selvaratnam, Krystle Amog, Shazia Siddiqui, and Susan Le, for formatting the paper.

Contributors JA coordinated the review, screened citations and full-text articles, abstracted data, appraised risk of bias, cleaned the data, interpreted the results and wrote the manuscript. WZ co-coordinated the review, screened citations and fulltext articles, abstracted data, appraised risk of bias, cleaned the data and edited the manuscript. BP helped conceptualise the research, interpreted the results and edited the manuscript. VN, RC, JDI and MG screened citations and full-text articles, abstracted data, appraised risk of bias and edited the manuscript. SLB helped conceive the study and edited the manuscript. SES conceived the study, designed the study, obtained the funding, interpreted the results and edited the manuscript. ACT conceived the study, designed the study, obtained the funding, interpreted the results and wrote some of the manuscript. All authors approved the final version to be published.

Funding This work was supported by the South Africa WHO Country Office. SES is funded by a Tier 1 Canada Research Chair in Knowledge Translation and ACT is funded by a Tier 2 Canada Research Chair in Knowledge Synthesis.

Competing interests None declared.

Patient consent Not required.

Provenance and peer review Not commissioned; externally peer reviewed.

Data sharing statement The datasets used and/or analysed during the current study are available from the corresponding author upon reasonable request.

Open access This is an open access article distributed in accordance with the Creative Commons Attribution Non Commercial (CC BY-NC 4.0) license, which permits others to distribute, remix, adapt, build upon this work non-commercially, and license their derivative works on different terms, provided the original work is properly cited and the use is non-commercial. See: http://creativecommons.org/ licenses/by-nc/4.0/

(c) Article author(s) (or their employer(s) unless otherwise stated in the text of the article) 2018. All rights reserved. No commercial use is permitted unless otherwise expressly granted.

\section{REFERENCES}

1. Kohn LT, Corrigan JM, Donaldson MS. To err is human: building a Safer Health System. 6: National Academies Press, 2000.

2. World Health Organization. Guide for developing national patient safety policy and strategic plan. 2014. 
3. Pronovost PJ, Holzmueller CG, Ennen CS, et al. Overview of progress in patient safety. Am J Obstet Gynecol 2011;204:5-10.

4. American College of Obstetricians Gynecologists. Patient safety in obstetrics and gynecology. ACOG Committee Opinion No. 447. Obstet Gynecol 2009;114:1424-7.

5. Pettker CM, Thung SF, Lipkind HS, et al. A comprehensive obstetric patient safety program reduces liability claims and payments. Am J Obstet Gynecol 2014;211:319-25.

6. National Conference of State Legislatures. Health cost containment and efficiencies. Washington, D.C.: National Conference of State Legislatures, 2011.

7. Knox GE, Simpson KR, Garite TJ. High reliability perinatal units: an approach to the prevention of patient injury and medical malpractice claims. J Healthc Risk Manag 1999;19:24-32.

8. World Health Organization. Topic 1: What is patient safety. In: WHO Patient safety curriculum guide for medical schools. Geneva: World Health Organization, 2010.

9. AHRQ. Patient safety. https://psnet.ahrq.gov/glossary/p

10. Craig P, Dieppe P, Macintyre S, et al. Developing and evaluating complex interventions: the new Medical Research Council guidance. BMJ 2008;337:a1655

11. Medical Research Council. Developing and evaluating complex interventions: new quidance, 2008

12. Cardoso R, Zarin W, Nincic V, et al. Evaluative reports on medical malpractice policies in obstetrics: a rapid scoping review. Syst Rev 2017;6:181

13. Khangura S, Konnyu K, Cushman R, et al. Evidence summaries: the evolution of a rapid review approach. Syst Rev 2012;1:1(1):1.

14. Moher D, Liberati A, Tetzlaff J, et al. Preferred reporting items for systematic reviews and meta-analyses: the PRISMA statement. Ann Intern Med 2009;151:264-9.

15. Haynes RB, McKibbon KA, Wilczynski NL, et al. Optimal search strategies for retrieving scientifically strong studies of treatment from Medline: analytical survey. BMJ 2005;330:1179.

16. McGowan J, Sampson M, Salzwedel DM, et al. PRESS Peer Review of Electronic Search Strategies: 2015 Guideline Statement. J Clin Epidemiol 2016;75:40-6.

17. Knowledge Translation Program. Synthesi.SR. Toronto, Canada: Knowledge Translation Program, St. Michael's Hospital, 2012.

18. Higgins JP, Altman DG, Gøtzsche PC, et al. The Cochrane Collaboration's tool for assessing risk of bias in randomised trials. BMJ 2011;343:d5928.

19. Althabe F, Belizán JM, Villar J, et al. Mandatory second opinion to reduce rates of unnecessary caesarean sections in Latin America: a cluster randomised controlled trial. Lancet 2004;363:1934-40.
20. Althabe F, Buekens P, Bergel E, et al. A behavioral intervention to improve obstetrical care. N Engl J Med 2008;358:1929-40.

21. Chaillet N, Dumont A, Abrahamowicz M, et al. A cluster-randomized trial to reduce cesarean delivery rates in Quebec. N Engl J Med 2015;372:1710-21.

22. Colbourn T, Nambiar B, Bondo A, et al. Effects of quality improvement in health facilities and community mobilization through women's groups on maternal, neonatal and perinatal mortality in three districts of Malawi: MaiKhanda, a cluster randomized controlled effectiveness trial. Int Health 2013:5:180-95.

23. Dumont A, Fournier P, Abrahamowicz M, et al. Quality of care, risk management, and technology in obstetrics to reduce hospital-based maternal mortality in Senegal and Mali (QUARITE): a clusterrandomised trial. Lancet 2013;382:146-57.

24. Horbar JD, Carpenter JH, Buzas J, et al. Collaborative quality improvement to promote evidence based surfactant for preterm infants: a cluster randomised trial. BMJ 2004;329:1004.

25. Lumley J, Donohue L. Aiming to increase birth weight: a randomised trial of pre-pregnancy information, advice and counselling in inner-urban Melbourne. BMC Public Health 2006:6:299.

26. Nielsen PE, Goldman MB, Mann S, et al. Effects of teamwork training on adverse outcomes and process of care in labor and delivery: a randomized controlled trial. Obstet Gynecol 2007;109:48-55.

27. Olds DL, Kitzman H, Knudtson MD, et al. Effect of home visiting by nurses on maternal and child mortality: results of a 2-decade followup of a randomized clinical trial. JAMA Pediatr 2014;168:800-6.

28. Riley W, Davis S, Miller K, et al. Didactic and simulation nontechnical skills team training to improve perinatal patient outcomes in a community hospital. Jt Comm J Qual Patient Saf 2011;37:357-64.

29. Zongo A, Dumont A, Fournier P, et al. Effect of maternal death reviews and training on maternal mortality among cesarean delivery: post-hoc analysis of a cluster-randomized controlled trial. Eur $J$ Obstet Gynecol Reprod Biol 2015;185:174-80.

30. Shepperd S, Lewin S, Straus S, et al. Can we systematically review studies that evaluate complex interventions? PLOS Med 2009;6:e1000086.

31. Milne JK, Walker DE, Vlahaki D. Reflections on the Canadian MORE(OB) obstetrical risk management programme. Best Pract Res Clin Obstet Gynaecol 2013;27:563-9.

32. Colbourn T, Pulkki-Brännström AM, Nambiar B, et al. Costeffectiveness and affordability of community mobilisation through women's groups and quality improvement in health facilities (MaiKhanda trial) in Malawi. Cost Eff Resour Alloc 2015;13:1. 